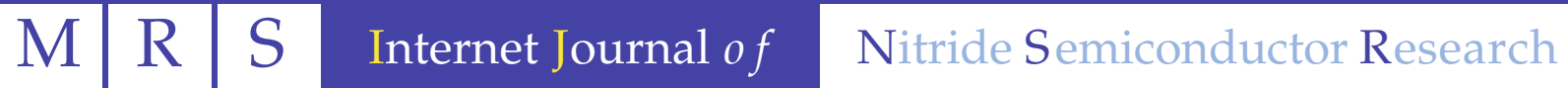

\author{
Volume 2, Article 46
}

\section{Characteristics of an Electron Cyclotron Resonance Plasma Source for the Production of Active Nitrogen Species in III-V Nitride Epitaxy}

\author{
M. Meyyappan \\ NASA Ames Research Center \\ This article was received on November 25, 1997 and accepted on December 11, 1997.
}

\begin{abstract}
A simple analysis is provided to determine the characteristics of an electron cyclotron resonance (ECR) plasma source for the generation of active nitrogen species in the molecular beam epitaxy of III-V nitrides. The effects of reactor geometry, pressure, power, and flow rate on the dissociation efficiency and ion flux are presented. Pulsing the input power is proposed to reduce the ion flux.
\end{abstract}

\section{Introduction}

The nitride family of materials cover an energy gap range of $1.9-6.2 \mathrm{eV}$ and exhibit high surface acoustic velocity, thermal conductivity, and high temperature stability. A wide variety of applications for the nitrides has been advocated: UV detectors, UV and visible LEDs, laser diodes for read-write applications, Bragg reflectors and wave guides, negative electron affinity cold cathodes, and transistors for high temperature operation. Potential also exists for miniaturization of on-board data storage for future generation unmanned space crafts, detection of chemical species with absorption bands in the blue/UV range, and cockpit displays. Molecular beam epitaxy (MBE) is one of the techniques to grow nitride layers with desirable electrical, structural and luminescent characteristics. This approach needs a source of active nitrogen species, and electron cyclotron resonance (ECR) plasma source has been popular in the nitride community [1] [2] [3] [4] [5] [6]. ECR source is widely used in silicon integrated circuit fabrication to deposit or etch semiconductor and insulating materials. It provides plasma densities of the order of 10 $11 \mathrm{~cm}^{-3}$ which is two orders of magnitude higher than that possible with radio frequency (rf) capacitive plasma sources. Such a high plasma density enables high rates of generation of active neutral species for materials processing applications. However, a large flux of ions may not be desirable for some applications, particularly for the growth of GaN [1], [2]. Hence, the nitride community is interested in large fluxes of active nitrogen species while keeping the flux of ions low. In this article, a model for the ECR nitrogen source is used to investigate the effects of various system parameters on the dissociation efficiency and ion densities.

\section{Analysis}

The operation of an ECR source is extremely complex and characterized by a strong coupling of various features: power coupling, magnetic field-microwave interaction, plasma generation, plasma heating, rarefied gas flow, generation and transport of active species, and interaction of various species with walls and wafers. Kinetic models such as particle-in-cell techniques to study the physics of plasma sources are complex. Recently, simple zero-dimensional models have been used to analyze high density plasmas used in processing applications [7] [8]. A 0-d model simply consists of balance equations for total mass, mass of various neutral and ionic species, and gas energy along with a plasma power (or energy) balance equation. The mass balance equations include, in addition to the inflow and outflow terms, all electron impact and thermal reaction terms as well as the effect of surface reactions as source or sink terms. The plasma power balance includes power spent on accelerating ions and power deposited to electrons; the latter is spent on elastic and inelastic collision and also lost due to wall recombination. The simplicity of the approach does not permit investigation of microwave-magnetic field interaction and related details; rather, the ECR power is just a lumped input parameter. The model provides volume-averaged densities of all species, gas, and electron temperatures. Obviously, no information on plasma uniformity or profiles can be obtained from such a global model. A computer code called SAMPR was used to conduct the analysis. For further details on the model, the reader is referred to [7] [8]. 
Electron impact rotational, vibrational, and electronic excitation of $\mathrm{N}_{2}$, ionization, dissociative ionization, and dissociation are included in this study. Neutral-neutral collisions have been ignored since the operating pressure in ECR devices is in the millitorr range. The wall collision of ions is assumed to liberate the corresponding neutral with a sticking probability of unity. The sticking coefficient for $\mathrm{N}$ atom recombination with stainless steel walls is taken to be 0.1 .

\section{ECR nitrogen discharge characteristics}

In this section, results from a systematic parametric study are presented to illustrate the effects of varying pressure, power, flow rate, and reactor dimensions. The basis case is chosen to correspond to the following conditions: source diameter $(d)=2.5 \mathrm{~cm}$, source length $(L)=10 \mathrm{~cm}$, pressure $(p)=1 \mathrm{mTorr}$, ECR power $(P)=30 \mathrm{~W}$, and $\mathrm{N}_{2}$ flow rate $(\mathrm{f})=10 \mathrm{sccm}$. The reactor dimensions correspond to a compact ECR source appropriate to fit onto a flange leading to the MBE growth chamber. It is assumed that the pressure and flow rate can be independently fixed at desirable values by adjusting the pumping speed / throttle valve. Note that the pressure is likely to vary along the length of the reactor under low pressure conditions $(<5 \mathrm{mTorr})$ since the pressure drop required to push the required mass flow through the system may be large. Such a variation cannot be accounted for in the present analysis.

Though coupled mass and energy balance equations are solved numerically in the present analysis, simple relations can be written below just for illustration purposes. Ignoring dissociative ionization of $\mathrm{N}_{2}$, a mass balance for nitrogen atoms considering flow through the system, generation through $\mathrm{N}_{2}$ dissociation, and loss due to wall recombination provides:

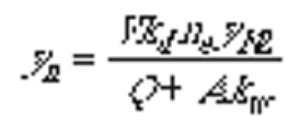

Here $y_{n}$ and $y_{N 2}$ are mass fractions of atomic and molecular nitrogen. One may use $y_{N 2}=1-y_{N}$ with a little error incurring due to the presence of vibrational and excitational populations. $V$ and $A$ are the ECR source volume and area respectively. $\mathrm{k}_{\mathrm{d}}$ (in $\mathrm{cm}^{3} / \mathrm{s}$ ) and $\mathrm{k}_{\mathrm{w}}$ (in $\mathrm{cm} / \mathrm{s}$ ) are rate coefficients for $\mathrm{N}_{2}$ dissociation and $\mathrm{N}$ atom wall recombination respectively. $Q$ is the flow rate at reactor conditions (and not the sccm value from the mass flow controller). $\eta_{e}$ is the electron density. Note that in a quasineutral plasma, $\mathrm{n}_{\mathrm{e}}=\mathrm{N}_{2}{ }^{+}$, ignoring the small population of $\mathrm{N}^{+}$ions.

The second useful relation is for the electron temperature which can be obtained from equating the ionization and ambipolar wall loss terms.

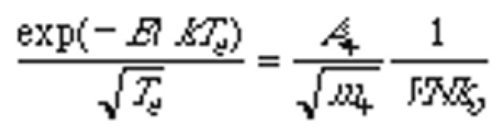

Here $E$ is the threshold energy for $\mathrm{N}_{2}$ ionization and $\mathrm{k}_{\mathrm{o}}$ is the pre-exponential factor in the Arrhenius relation. $\mathrm{k}$ is Boltzmann Constant and $T_{e}$ is the electron temperature. $m_{+}$is the mass of $N_{2}{ }^{+} . A_{+}$is the effective area for ion loss [7] [8]. $\mathrm{N}$ is the number density of molecular nitrogen.

Figure 1 shows the $\mathrm{N}_{2}{ }^{+}$density and mass fractions of atomic nitrogen as a function of pressure with all other parameters at their base values. As pressure increases, ion density increases rapidly at first and slower at higher pressures. Atomic nitrogen fraction increases until $1 \mathrm{mTorr}$ and decreases at higher pressures. In the relation for atomic nitrogen fraction, the wall recombination is independent of pressure and hence, the behavior is dictated by the variation of $Q$ and the product of $k_{d} n_{e}$. The volumetric flow rate at reactor conditions, for a fixed $10 \mathrm{sccm}$, goes down as pressure increases. Note that an increase in pressure results in a decrease in electron temperature according to the second relation which would reduce the value of dissociation rate constant, $k_{d}$. At pressures above 1 mtorr, the product $k_{d} n_{e}$ decreases significantly and is responsible for the observed behavior of $\mathrm{N}$ atom mass fraction vs. pressure.

The effect of ECR source power is seen from Table 1. The ion density increases rapidly with power. There is also a moderate variation in electron temperature due to the changes in molecular nitrogen density following dissociation. 
The fraction of nitrogen atoms reaches $25 \%$ at $60 \mathrm{~W}$. The flux of nitrogen atoms, obtained by the product of total mass flow and nitrogen atom mass fraction and using the source cross sectional area, is $1-5 \times 10^{17} \mathrm{~cm}^{-2} \mathrm{~s}^{-1}$.

The effect of $\mathrm{N}_{2}$ flow rate is presented in Table 2 with all other variables kept at their base values. There is a moderate change in the ion density with the flow rate. Since the residence time of the gas decreases with an increase in flow rate, the production of $\mathrm{N}$ atoms and atomic nitrogen density are reduced but the flux still increases due to an increase in mass flow.

The effect of increasing the ECR source diameter was also examined with all other variables under their base conditions. Note that power density decreases with an increase in the size of the chamber. This reduces the ion density by a factor of two when the diameter changes from 2.5 to $7.5 \mathrm{~cm}$. There is a corresponding reduction in the atomic nitrogen density also from $7.3 \times 10^{12} \mathrm{~cm}^{-3}$ for $\mathrm{d}=2.5 \mathrm{~cm}$ to $5.14 \times 10^{12}$ for $\mathrm{d}=7.5 \mathrm{~cm}$.

\section{Pulsed-power nitrogen discharge}

From the discussion in the previous section, it is clear that an ECR discharge is capable of high $\mathrm{N}_{2}$ dissociation efficiency and yielding a large flux of atomic nitrogen. However, this is inevitably accompanied by ion densities well in the mid- $10^{10}$ to $10^{11} \mathrm{~cm}^{-3}$ range. Further calculations reveal that addition of an inert gas such as argon $(0-50 \%)$ does not provide any relief either. In this section, the effect of pulsing the source power is discussed. Pulsed-power plasmas have been recently proposed to eliminate anomalous side wall etching problems in high density discharges and characteristics of an $\mathrm{SF}_{6}$ pulsed ECR discharge have been discussed in ref. [9].

Figure 2 shows the effect of pulsing the ECR source power for a pulse period of $100 \mu \mathrm{s}(10 \mathrm{KHz})$ with all parameters fixed at their base values. A simple square wave modulation of the input power is considered. Note that the pulse period is the sum of the on-and off- time (i.e. pulse width + pulse interval). Three duty ratios (i.e. ratio of on-time to total period) of $0.25,0.5$, and 0.75 are examined; for a power of $30 \mathrm{~W}$, the time-averaged power in each case would be $7.5,15.0$, and $22.5 \mathrm{~W}$ respectively. Soon after the power is turned on, the electron energy rises rapidly to very high values due to the small electron population and decreases to normal values during the on-period. The electron energy is negligible when the power is off and consequently, all electron impact reactions are negligible during this time. The physics behind the observations in Figure 2 has been explained in ref. [9]. The important point of interest here is the ion density steadily drops during the off-period. The steady decrease is the strongest for a duty ratio of 0.25 though the peak during power-on is slightly higher than that at duty $=0.5$ and 0.75 . The time-averaged ion density is $3.9 \times 10^{10}, 5.8 \times 10^{10}$, and $7.1 \times 10^{10} \mathrm{~cm}^{-3}$ for duty $=0.25,0.5$, and 0.75 respectively. The corresponding result for the $\mathrm{CW}$ operation is $7.9 \times 10^{10} \mathrm{~cm}^{-3}$. There is a factor of two reduction in ion density for $25 \%$ duty compared to $\mathrm{CW}$ operation. However, the atomic nitrogen density decreases to $2.1 \times 10^{12}$ from $7.3 \times 10^{12} \mathrm{~cm}^{-3}$. Note that the power level is reduced by a factor of four from $30 \mathrm{~W}$ to a time-averaged $7.5 \mathrm{~W}$. It appears then pulsing the power provides an avenue to reduce the ion flux while maintaining reasonable atomic nitrogen flux. It is clear from Figure 2 that the atomic nitrogen output is hardly modulated by the pulse which is important for uninterrupted growth. Finally, pulse periods of 50 to $200 \mu$ s have been examined and found to display similar behavior as in Figure 2. However, for a given duty ratio, the longer pulse period gives smaller time-averaged ion flux as well as atomic nitrogen flux.

\section{Concluding Remarks}

ECR and similar high density sources utilize the input power more efficiently than rf capacitive sources. In the latter case, a significant fraction of the power goes to accelerating the ions in the sheath and consequently, the plasma density is in the $10^{9} \mathrm{~cm}^{-3}$ range. In contrast, power coupling in ECR at low pressures is more efficient and characterized by a plasma density $\sim 10^{11} \mathrm{~cm}^{-3}$. While this is good for the production of active species, a persistent high flux of ions is detrimental in GaN growth. Operation of a compact source at pressures about $1 \mathrm{mTorr}$ and low power levels $(\sim 30 \mathrm{~W})$ appears to provide ion densities in the mid $-10^{10} \mathrm{~cm}^{-3}$ range while providing a high flux of active nitrogen species (atomic and excited $\mathrm{N}_{2}$ ). This level of ion flux may still be high. Introduction of biased grids to extract ions may work but would significantly alter the operation and stability of a compact source. Pulsing the source power has been shown to provide a partial relief to the ion flux problem while still maintaining reasonable levels of active nitrogen species.

\section{References}


[1] R.J. Molnar, R. Singh, T.D. Moustakas, J. Electron. Mater. 24, 275 (1995).

[2] R.J. Molnar, T.D. Moustakas, J. Appl. Phys. 76, 4587 (1994).

[3] R. Beresford, A. Ohtani, K. S. Stevens, M. Kinniburgh, J. Vac. Sci. Technol. B 13, 792-795 (1995).

[4] W. T. Tafemer, A. Bensaoula, E. Kim, A. Bousetta, J. Vac. Sci. Technol. B 14, 2357 (1996).

[5] U. Rossner, D. Brun-Le Cunff, A. Barski, B. Daudin, J. Vac. Sci. Technol. A 14, 2655 (1996).

[6] S. Sinharoy, G. Augustine, L. B. Rowland, A. K. Agarwal, R. L. Messham, M. C. Driver, R. H.Hopkins ,J. Vac. Sci. Technol. A 14, 896-899 (1996).

[7] M. Meyyappan, T. R. Govindan, IEEE Trans. Plasma Sci. 23, 623 (1995).

[8] M. Meyyappan, T. R. Govindan, Vacuum 47, 215 (1996).

[9] M. Meyyappan, Jpn. J. Appl. Phys. 36, 4820 (1997).

\section{Table 1}

Effect of ECR source power on ion density $\left(\mathrm{N}_{2}{ }^{+}\right)$, electron temperature $\left(\mathrm{T}_{e}\right)$, and mass fraction of atomic nitrogen. 1 mTorr, $10 \mathrm{sccm} \mathrm{N}_{2}$ flow, $\mathrm{d}=2.5 \mathrm{~cm}$, and $\mathrm{L}=10.0 \mathrm{~cm}$.

\begin{tabular}{|c||c||c||c||}
\hline \begin{tabular}{c||c||}
\hline Power \\
$(\mathrm{W})$
\end{tabular} & $\mathrm{N}_{2}{ }^{+}$ & $\mathrm{T}_{\mathrm{e}}$ & $\mathrm{y}_{\mathrm{N}}$ \\
& $(\mathrm{cm})$ & $(\mathrm{eV})$ & \\
\hline \hline 10 & $3.1 \times 10^{10}$ & 8.3 & $5.8 \%$ \\
\hline \hline 20 & $5.8 \times 10^{10}$ & 9.1 & $10.8 \%$ \\
\hline \hline 30 & $7.9 \times 10^{10}$ & 10.0 & $15.2 \%$ \\
\hline \hline 50 & $1.1 \times 10^{11}$ & 12.0 & $22.0 \%$ \\
\hline \hline 60 & $1.2 \times 10^{11}$ & 13.0 & $24.6 \%$ \\
\hline \hline
\end{tabular}

\section{Table 2}

Effect of $\mathrm{N}_{2}$ flow rate on ion density $\left(\mathrm{N}_{2}{ }^{+}\right)$, atomic nitrogen fraction $\left(\mathrm{y}_{\mathrm{N}}\right)$, its number density, and flux.

\begin{tabular}{|c||c||c||c||c||}
\hline \begin{tabular}{|c||c||c||}
$\mathrm{N}_{2}$ flow \\
$(\mathrm{Sccm})$
\end{tabular} & $\mathrm{N}_{2}{ }^{+}$ & $\mathrm{y}_{\mathrm{N}}$ & $\begin{array}{c}\text { Atomic } \mathrm{N} \\
\left(\mathrm{cm}^{-3}\right)\end{array}$ & $\begin{array}{l}\text { Atomic flux } \\
\left(\mathrm{cm}^{-2} \mathrm{~s}^{-1}\right)\end{array}$ \\
\hline \hline 5 & $7.4 \times 10^{10}$ & $20.0 \%$ & $9.2 \times 10^{12}$ & $1.8 \times 10^{17}$ \\
\hline \hline 10 & $7.9 \times 10^{10}$ & $15.2 \%$ & $7.3 \times 10^{12}$ & $2.8 \times 10^{17}$ \\
\hline \hline 15 & $8.2 \times 10^{10}$ & $12.3 \%$ & $6.0 \times 10^{12}$ & $3.4 \times 10^{17}$ \\
\hline \hline 20 & $8.5 \times 10^{10}$ & $10.4 \%$ & $5.2 \times 10^{12}$ & $3.8 \times 10^{17}$ \\
\hline \hline 100 & $8.8 \times 10^{10}$ & $3.0 \%$ & $1.6 \times 10^{12}$ & $5.5 \times 10^{17}$ \\
\hline \hline
\end{tabular}




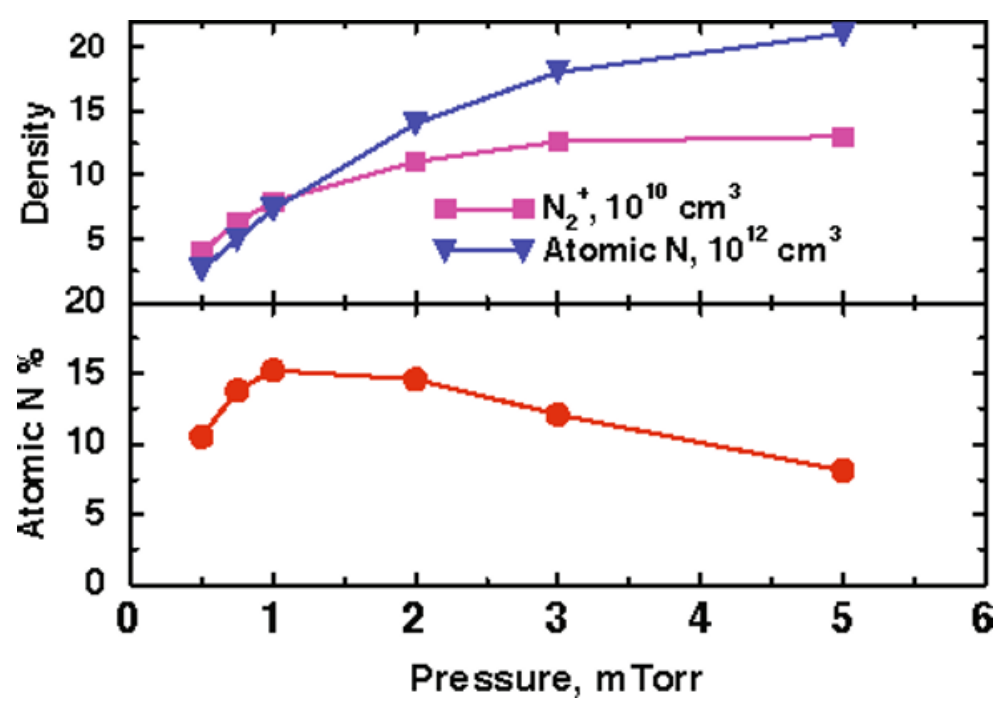

Figure 1. Effect of pressure on the ECR discharge characteristics. Source diameter $=2.5$ $\mathrm{cm}$ and length $=10 \mathrm{~cm} .10 \mathrm{sccm}$ of $\mathrm{N}_{2}$ at $30 \mathrm{~W}$ power.

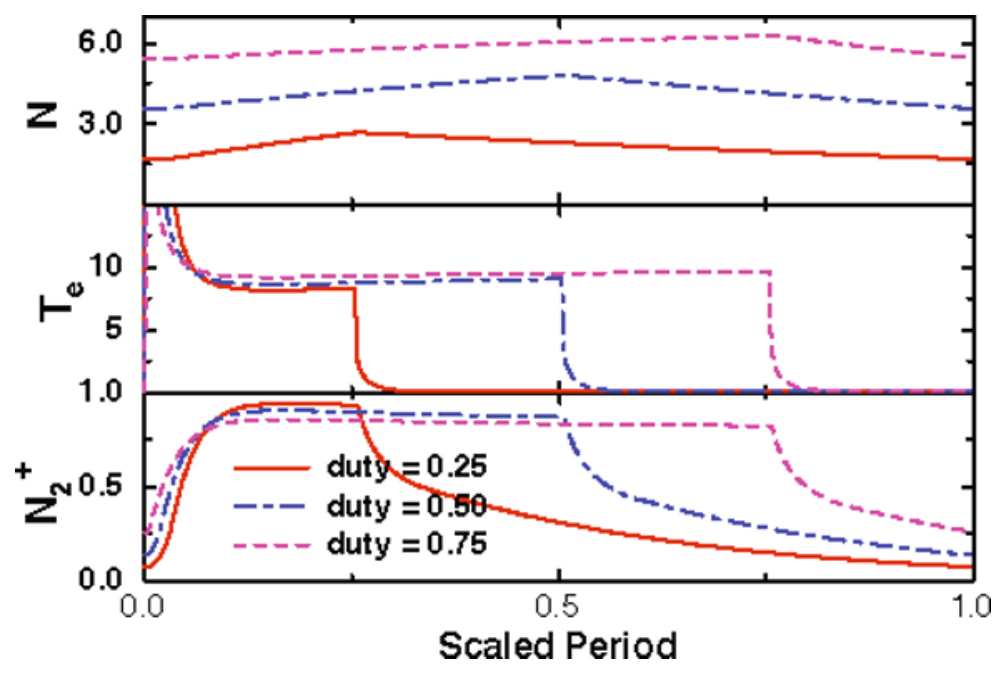

Figure 2. Effect of pulsing the ECR power. Pulse period $=100 \mu \mathrm{s}$. Three duty ratios of $25 \%, 50 \%$ and $75 \%$ are examined. Discharge conditions: 1 mTorr, 10 sccm of $\mathrm{N}_{2}$ and $30 \mathrm{~W}$ power. Top: atomic nitrogen density in $10^{12} \mathrm{~cm}^{-3}$; middle: electron temperature in $\mathrm{eV}$; and bottom: $\mathrm{N}_{2}{ }^{+}$ density in $10^{11} \mathrm{~cm}^{-3}$.

(C) 1997 The Materials Research Society

\begin{tabular}{|l|l|l|l|l}
\hline M & $\mathrm{R}$ & $\mathrm{S}$ & Internet Journal of & Nitride Semiconductor Research \\
\hline
\end{tabular}

